Article

\title{
Open Gromov-Witten Invariants from the Augmentation Polynomial
}

\author{
Matthew Mahowald ${ }^{+}$ \\ Department of Mathematics, Northwestern University, Evanston, IL 60208, USA; \\ m.mahowald@u.northwestern.edu \\ † Current address: 222 S Riverside Plaza Ste 2800, Chicago, IL 60606, USA
}

Received: 30 August 2017; Accepted: 10 October 2017; Published: 17 October 2017

\begin{abstract}
A conjecture of Aganagic and Vafa relates the open Gromov-Witten theory of $X=\mathcal{O}_{\mathbb{P}^{1}}(-1,-1)$ to the augmentation polynomial of Legendrian contact homology. We describe how to use this conjecture to compute genus zero, one boundary component open Gromov-Witten invariants for Lagrangian submanifolds $L_{K} \subset X$ obtained from the conormal bundles of knots $K \subset S^{3}$. This computation is then performed for two non-toric examples (the figure-eight and three-twist knots). For $(r, s)$ torus knots, the open Gromov-Witten invariants can also be computed using Atiyah-Bott localization. Using this result for the unknot and the $(3,2)$ torus knot, we show that the augmentation polynomial can be derived from these open Gromov-Witten invariants.
\end{abstract}

Keywords: mirror symmetry; Gromov-Witten theory; Legendrian contact homology; knot theory

\section{Introduction}

Gromov-Witten theory has famously benefited from its connections with string dualities, first with mirror symmetry [1,2], and more recently, large $N$ duality [3,4]. Beginning with [5], for toric manifolds, Gromov-Witten invariants associated with maps of closed surfaces have also been systematically computed using localization [6-8]. Although the analogous constructions in open Gromov-Witten theory are not rigorously defined in general, many of the same computational tools (such as mirror symmetry and Atiyah-Bott localization) can still be applied. In contrast to the closed theory, open Gromov-Witten theory also possesses many direct relationships with knot theory. Large $N$ duality relates Chern-Simons theory on $S^{3}$ to Gromov-Witten theory on $X=\mathcal{O}_{\mathbb{P} 1}(-1,-1)$ via the conifold transition. Wilson loops in Chern-Simons theory on $S^{3}$ are also related to the HOMFLY polynomials of knots $K \subset S^{3}$ [9]. This relationship equates the colored HOMFLY polynomials of knots $K$ with generating functions for open Gromov-Witten invariants of $X$ with Lagrangian boundary $L_{K}$ obtained from the conormal bundle $N_{K}^{*} \subset T^{*} S^{3}$, and has been checked for torus knots in [10].

Recently, it has also been suggested that open Gromov-Witten theory is related to another type of knot invariant arising in Legendrian contact homology [11,12]. For a knot $K \subset S^{3}$, Legendrian contact homology associates a dga $\mathcal{A}\left(\Lambda_{K}\right)$ with its unit conormal bundle $\Lambda_{K} \subset U^{*} S^{3}$. The unit conormal bundle $\Lambda_{K} \approx T^{2}$ is a Legendrian submanifold of the unit cotangent bundle $U^{*} S^{3}$ (a contact manifold), and the differential on $\mathcal{A}\left(\Lambda_{K}\right)$ is obtained, roughly speaking, from counts of maps of holomorphic disks to $\Lambda_{K} \times \mathbb{R}$. An augmentation of $\mathcal{A}\left(\Lambda_{K}\right)$ is a dga map $\epsilon: \mathcal{A}\left(\Lambda_{K}\right) \rightarrow \mathbb{C}$, where $\mathbb{C}$ is interpreted as a dga with trivial differential. The moduli space of such augmentations is described by an equation $A_{K}(x, p, Q)=0$, where $x, p$, and $Q$ are generators for $H_{2}\left(U^{*} S^{3}, \Lambda_{K}\right) . A_{K}$ is called the augmentation polynomial of the knot $K$ (more detailed accounts of Legendrian contact homology can be found in $[12,13])$.

Mirror symmetry is a relationship between two Calabi-Yau manifolds $X$ and $\hat{X}$ that identifies the symplectic structure on $X$ with the complex structure on $\hat{X}$, and vice versa $($ see $[1,2])$. 
The Strominger-Yau-Zaslow conjecture offers a geometric interpretation of mirror symmetry in terms of dual torus fibrations by special Lagrangian submanifolds [14]. When $X$ is $\mathcal{O}_{\mathbb{P}^{1}}(-1,-1)$, the special Lagrangian fibers can be understood as originating from conormal bundles to unknots $K \subset S^{3}$ under large $N$ duality [3,4]. In [11], Aganagic and Vafa generalize the Strominger-Yau-Zaslow conjecture to cases where the special Lagrangian fibers are obtained from arbitrary knots $K \subset S^{3}$ by identifying this moduli space with the augmentation polynomial $A_{K}$ of the knot. Mirror symmetry interprets this moduli space as the superpotential associated with the B-model topological string theory. The dual A-model theory's superpotential contains the open Gromov-Witten invariants associated with $L_{K}$, so Aganagic and Vafa's conjecture can be rephrased as follows:

Conjecture 1. Let $K \subset S^{3}$ be a knot, and $L_{K} \subset X$ be a Lagrangian submanifold of $X=\mathcal{O}_{\mathbb{P}^{1}}(-1,-1)$ with geometry $K \times \mathbb{R}^{2}$. Let $A_{K}$ denote the augmentation polynomial of $K$, and $W_{K}(x, Q)$ denote the genus-zero, one boundary component open Gromov-Witten potential

$$
W_{K}(x, Q)=\sum_{d, w} K_{d, w}^{0,1} Q^{d} x^{w}
$$

Then,

$$
\int-\log p(x, Q) \frac{d x}{x}=W_{K}(x, Q),
$$

where $p(x, Q)$ is the function implicitly defined by the equation $A_{K}(x, p, Q)=0$. More generally, the moduli of Lagrangian branes with geometry $K \times \mathbb{R}^{2}$ is given by the equation

$$
A_{K}(x, p, Q)=0,
$$

and $K$ determines a mirror manifold $\hat{X}_{K}$ defined by the hypersurface

$$
\hat{X}_{K}=\left\{u v=A_{K}(x, p, Q)\right\} \subset \mathbb{C}^{4} .
$$

The goal of this paper is to use this conjecture to compute Gromov-Witten invariants and augmentation polynomials. Because the augmentation polynomial can be computed for non-toric knots, this method can be applied in scenarios where Atiyah-Bott localization cannot be used. On the other hand, for $(r, s)$ torus knots, open Gromov-Witten invariants are known from localization, and this data provides another means of obtaining the augmentation polynomial of $K$.

\subsection{Statement of Main Results}

The main result of this paper is a technique for computing open Gromov-Witten invariants using Conjecture 1. This technique is applied for two non-toric knots (the $4_{1}$ and $5_{2}$ knots) for which a direct approach using Atiyah-Bott localization is intractable.

Proposition 1. Let $K_{d, w}$ denote the open Gromov-Witten invariants of degree $d$ and winding $w$ for the $4_{1}$ and $5_{2}$ knots in framing 0 computed using Conjecture 1 . Then, for $w \leq 8$ and all $d, K_{d, w}$ satisfy the integrality constraint

$$
K_{d, w}=\sum_{n \mid d \text { and } n \mid w} \frac{1}{n^{2}} N_{d / n, w / n} .
$$

While, for non-toric knots, $K_{d, w}$ cannot be found through localization (removing the possibility of comparison against direct computation), the integrality constraint (2) is a strong restriction satisfied by Gromov-Witten invariants. For a toric knot, the situation is better-a comparison with direct calculation can be made. This is done for two simple knots (the unknot and $(3,2)$ trefoil knot), and can (at least in theory) be done for any torus knot. 
Proposition 2. Let $K_{d, w}$ denote the open Gromov-Witten invariants of degree $d$ and winding $w$ for the unknot and $(3,2)$ torus knot. Then, for each knot, the augmentation polynomial $A_{K}$ can be recovered from the corresponding open Gromov-Witten invariants.

Note that, for an $(r, s)$ torus knot, the computational complexity of the algorithm used for this calculation scales quite poorly with increasing $r, s$, so there are practical limitations to obtaining augmentation polynomials for arbitrary torus knots in this way.

\subsection{Organization of the Paper}

This paper is organized in the following way. Section 2 reviews mirror symmetry for open Gromov-Witten invariants, and describes Aganagic and Vafa's conjecture relating mirror symmetry and the augmentation polynomial. Section 3 applies this conjecture to compute open Gromov-Witten invariants in two non-toric examples. Finally, Section 4 performs the reverse of this computation: the open Gromov-Witten invariants associated to torus knots are used to recover the corresponding augmentation polynomials. (Note that, for $(r, s)$ torus knots, open Gromov-Witten invariants in framing $r s$ have been computed directly via localization in [10].)

\section{Open String Mirror Symmetry and the Augmentation Polynomial}

Recently, new developments in knot theory and open topological string theory have uncovered connections between open Gromov-Witten theory and knot theory [4,10-12]. The subject of interest in this note is open Gromov-Witten theory for Lagrangian submanifolds of $X=\mathcal{O}_{\mathbb{P}^{1}}(-1,-1)$. Let $\Sigma$ be a genus-zero Riemann surface with one boundary component. Denote by $K_{d, w}$ the open Gromov-Witten invariant associated with a stable map $f: \Sigma \rightarrow X$ with Lagrangian boundary conditions on a Lagrangian submanifold $L \subset X$, where $d=[f(\Sigma)] \in H_{2}(X, L)$ and $w=[f(\partial \Sigma)] \in H_{1}(L)$. This section describes a technique for computing $K_{d, w}$ using a mirror symmetry conjecture [11,12].

$X$ can be obtained by symplectic reduction on $\mathbb{C}^{4}$. Let $\left(z_{1}, z_{2}, z_{3}, z_{4}\right)$ be coordinates for $\mathbb{C}^{4}$, and let $S^{1}$ act on $\mathbb{C}^{4}$ with weights $(1,1,-1,-1)$; then,

$$
X \cong\left\{\left|z_{1}\right|^{2}+\left|z_{2}\right|^{2}-\left|z_{3}\right|^{2}-\left|z_{4}\right|^{2}=r\right\} / S^{1},
$$

where $r \in \mathbb{R}_{>0}$. The coordinates on the base $\mathbb{P}^{1}$ are $z_{1}$ and $z_{2}$, and the $z_{3}, z_{4}$ coordinates parametrize the fiber. According to the Strominger-Yau-Zaslow conjecture for noncompact $X[14,15], X$ is a special Lagrangian fibration over a base $B \cong \mathbb{R}^{3}$, with generic fibers $L \cong T^{2} \times \mathbb{R}$. In these coordinates, the base $B$ and the special Lagrangian fibers $L$ are easy to describe. The base $B$ is the image of $X$ under the moment map $z_{i} \mapsto\left|z_{i}\right|^{2}$, and the fibers $L$ are given by the equations

$$
\begin{aligned}
\left|z_{2}\right|^{2}-\left|z_{4}\right|^{2} & =c_{1}, \\
\left|z_{3}\right|^{2}-\left|z_{4}\right|^{2} & =c_{2}, \\
\operatorname{Arg}\left(z_{1} z_{2} z_{3} z_{4}\right) & =0,
\end{aligned}
$$

where $c_{1}, c_{2} \in \mathbb{R}$. For generic values of $c_{1}, c_{2}, L$ has topology $T^{2} \times \mathbb{R}$; however, along a critical locus (such as when either $c_{1} \rightarrow 0$ or $c_{2} \rightarrow 0$ ), the topology of the fibers degenerates to two copies of $S^{1} \times \mathbb{R}^{2}$. This critical locus along which the fibers degenerate forms a trivalent graph in $B$, corresponding to the "edges" of the moment polytope. The moment polytope and special Lagrangian fibers are depicted in Figure 1. 


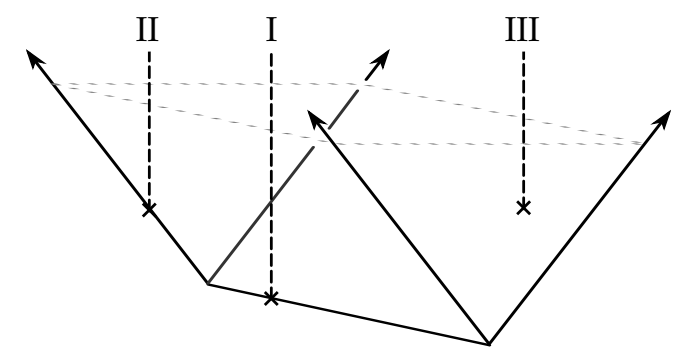

Figure 1. The moment polytope and special Lagrangian fibers of $X$. The moment polytope of $X=\mathcal{O}_{\mathbb{P}^{1}}(-1,-1)$ is its image $\pi(X) \subset \mathbb{R}^{4}$ under the moment map $\pi\left(z_{i}\right)=\left|z_{i}\right|^{2}$. The images of the special Lagrangian fibers of $X$ in the moment polytope are vertical lines, which can intersect $X$ three ways: along the base $\mathbb{P}^{1}$ (type I), along an exterior leg of the polytope (type II), or on a face (type III). Lagrangian fibers of type III have topology $T^{2} \times \mathbb{R}$, corresponding to nondegenerate solutions in (4). Fibers of type I and II have topology $S^{1} \times \mathbb{R}^{2}$, corresponding to degenerate solutions (such as $c_{2}=0$ or $c_{1}=0$, respectively).

\subsection{The Mirror of $\mathcal{O}_{\mathbb{P}^{1}}(-1,-1)$}

The construction of $[15,16]$ gives the mirror manifold $\hat{X}$ to $X$ in terms of a dual Landau-Ginsburg theory. For $X=\mathcal{O}_{\mathbb{P}^{1}}(-1,-1)$, the mirror geometry is obtained from a $U(1)$ linear sigma model of four fields with charges $(1,1,-1,-1)$. The mirror geometry is described by

$$
\hat{X}=\left\{u v=y_{2}+y_{3}+y_{4}+\frac{y_{3} y_{4}}{y_{2}} e^{-t}\right\},
$$

where $u, v \in \mathbb{C}, y_{j} \in \mathbb{C}^{*}, t=r+i \theta$ is the complexified Kähler parameter, and $y_{1}$ has been eliminated using the relation $y_{1} y_{2}=y_{3} y_{4} e^{-t}$. The Strominger-Yau-Zaslow (SYZ) conjecture [14] asserts that the mirror $\hat{X}$ is the moduli of the special Lagrangian fibers of $X$. The choice of coordinate patch for the $y_{j}$ 's determines which "phase" of Lagrangian fibers is being described. This coordinate choice is explained in further detail in [17]. In this note, the relevant coordinates are the $y_{2}=1$ patch. In addition, to achieve later agreement with conventions from knot contact homology, the following change of coordinates will be used:

$$
Q:=e^{t}, \quad x:=-y_{3} / Q, \quad p:=-y_{4} / Q .
$$

Then, in this patch and coordinate system, the mirror manifold is

$$
\hat{X}=\{u v=1-Q x-Q p+Q x p\}
$$

Example 1 (Open Gromov-Witten invariants via mirror symmetry). Consider Lagrangian fibers $L$ of type II. These Lagrangians have topology $S^{1} \times \mathbb{R}^{2}$, and intersect the base $\mathbb{P}^{1}$ along the $S^{1}$. In the mirror, the moduli space of such Lagrangian fibers is the Riemann surface $S \subset \hat{X}$ defined by setting $u v=0$ :

$$
S=\{1-Q x-Q p+Q x p=0\} .
$$

Mirror symmetry equates the periods of certain differential forms on $\hat{X}$ to generating functions for open Gromov-Witten invariants. In this case, the prediction of mirror symmetry is that (up to constant factors in $x$ )

$$
\int \lambda=\sum_{d, w} K_{d, w} Q^{d} x^{w}
$$


where $\lambda:=-\log p \frac{d x}{x}$ is a one-form along $S$ defined by solving $1-Q x-Q p+Q x p=0$ for $p$, and $K_{d, w}$ is the genus-0, degree-d, winding w open Gromov-Witten invariant with boundary on L. In terms of $x$ and $Q$,

$$
\begin{aligned}
-\log p(x ; Q) & =\log (Q)+\log \left(\frac{1-x}{1-Q x}\right) \\
& =\log (Q)+\sum_{n=1}^{\infty} \frac{1}{n}\left(-1+Q^{n}\right) x^{n},
\end{aligned}
$$

so Equation (6) asserts that

$$
\sum_{d, w} K_{d, w} Q^{d} x^{w}=\sum_{n=1}^{\infty} \frac{1}{n^{2}}\left(-1+Q^{n}\right) x^{n}
$$

Hence,

$$
K_{d, w}= \begin{cases}-\frac{1}{w^{2}}, & d=0, \\ \frac{1}{d^{2}}, & d=w, \\ 0, & \text { otherwise. }\end{cases}
$$

Note that, in this case, $K_{d, w}$ has also been computed directly using localization in [18], and these results agree in framing 0.

Open Gromov-Witten invariants are also conjectured to satisfy certain integrality requirements $[15,19]$. For genus-zero, one-boundary-component invariants, the requirement is that

$$
K_{d, w}=\sum_{n \mid d \text { and } n \mid w} \frac{1}{n^{2}} N_{d / n, w / n}
$$

where $N_{d, w} \in \mathbb{Z}$. In terms of the generating function for $K_{d, w}$, this is

$$
\sum_{d, w} K_{d, w} Q^{d} x^{w}=\sum_{n>0} \sum_{d, w} \frac{1}{n^{2}} N_{d, w} Q^{d} x^{w} .
$$

In the previous example, $N_{0,1}=-1, N_{1,1}=1$, and $N_{d, w}=0$ for all other $d, w$.

\subsection{Knots and the Conifold Transition}

The Lagrangian submanifolds described by Equation (4) have a very specific geometry. A natural question is: What are the open Gromov-Witten invariants associated with Lagrangians with a different geometry? One way of obtaining such Lagrangians is through the conifold transition $[4,10]$. The manifold $X=\mathcal{O}_{\mathbb{P}^{1}}(-1,-1)$ can also be obtained as the resolution of the conifold singularity in $\mathbb{C}^{4}$-it is given by the equations

$$
\begin{aligned}
x z-y w & =0, \\
x \lambda & =w \rho, \\
y \lambda & =z \rho,
\end{aligned}
$$

where $((x, y, z, w),[\lambda: \rho]) \in \mathbb{C}^{4} \times \mathbb{P}^{1}$. The conifold singularity $x z-y w=0$ is the limit of the hypersurface

$$
Y_{\mu}:=\{x z-y w=\mu\} \subset \mathbb{C}^{4}
$$

as $\mu \rightarrow 0$. For $\mu \neq 0, Y_{\mu}$ is symplectomorphic to the cotangent bundle $T^{*} S^{3}$. The zero section $S_{\mu} \cong S^{3} \subset Y_{\mu}$ is the fixed locus of the antiholomorphic involution $(x, y, z, w) \mapsto(\bar{z},-\bar{w}, \bar{x},-\bar{y})$, and is described by the equation $|x|^{2}+|y|^{2}=\mu$. Away from the zero sections, the conifold transition gives 
a map between $T^{*} S^{3}$ and $X$ [10]. Consequently, a Lagrangian submanifold of $T^{*} S^{3}$ that does not intersect the zero section $S^{3}$ can be identified with a Lagrangian submanifold of $X$, which also does not intersect the zero section.

Knots $K \subset S^{3}$ are a source of Lagrangian submanifolds in $T^{*} S^{3}$ : the conormal bundle $N_{K}^{*} \subset T^{*} S^{3}$ is Lagrangian with topology $S^{1} \times \mathbb{R}^{2}$. In order to obtain a Lagrangian submanifold of $X$ from $N_{K}^{*}, N_{K}^{*}$ must first be moved off of the zero section. This can be done by choosing a lift of $K$, as described in [10]. The image of the shifted conormal bundle will be a Lagrangian submanifold $L_{K} \subset X$, as depicted in Figure 2.

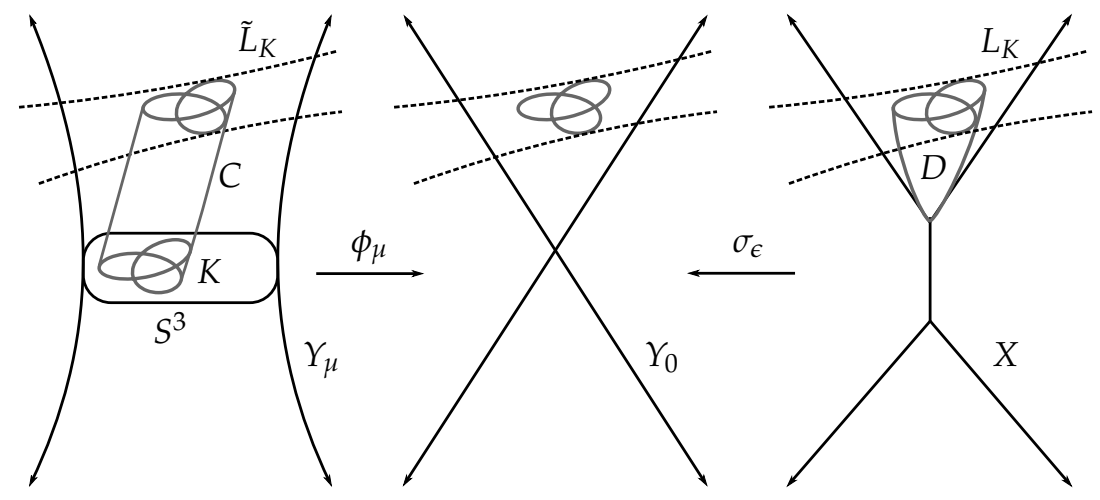

Figure 2. The conifold transition and knots. The Lagrangian $\tilde{L}_{K} \subset Y_{\mu} \cong T^{*} S^{3}$ is constructed by shifting the conormal bundle of a knot $K \subset S^{3}$ off of the zero section. This lift introduces a holomorphic cylinder $C$ connecting the knot on $S^{3}$ to its image in $\tilde{L}_{K} . Y_{0}$ is the conifold singularity $x z-y w=0$ in $\mathbb{C}^{4}$. The map $\phi_{\mu}: Y_{\mu} \rightarrow Y_{0}$ is a symplectomorphism away from the zero section, so $\phi_{\mu}\left(\tilde{L}_{K}\right)$ is a Lagrangian submanifold of $Y_{0}$. $X \cong \mathcal{O}_{\mathbb{P}^{1}}(-1,-1)$ is the small resolution of the conifold singularity, and $\sigma_{\epsilon}: X \rightarrow Y_{0}$ is the corresponding natural map. In fact, there are a family of such maps, where $\epsilon$ parametrizes the symplectic area of the zero section $\mathbb{P}^{1} \subset X$. Hence, $L_{K}:=\sigma_{\epsilon}^{-1} \circ \phi_{\mu}\left(\tilde{L}_{K}\right)$ is a Lagrangian submanifold of $X$. The holomorphic disk $D$ is the image of $C$ under the conifold transition.

\subsection{Open Gromov-Witten Invariants and the Augmentation Polynomial}

For torus knots, the corresponding open Gromov-Witten invariants of $\left(X, L_{K}\right)$ have been computed directly using localization in [10], and using mirror symmetry in [20,21]. However, the mirror symmetry approach of [20] does not readily generalize to non-toric knots. In this subsection, a recent conjecture of Aganagic and Vafa [11] is applied to compute open Gromov-Witten invariants via an analogous mirror symmetry computation. This approach uses the augmentation polynomial from Legendrian contact homology. In contrast to localization and the method of [20], this method can be applied for any knot whose augmentation polynomial is known-including non-toric knots.

The Aganagic-Vafa conjecture can be motivated by observing that the mirror to $\hat{X}$ given in Equation (5) can be written as

$$
\hat{X}=\{u v=A(x, p ; Q)\},
$$

where $A(x, p ; Q)=1-Q x-Q p+Q x p$ is the augmentation polynomial of the unknot in Legendrian contact homology $[13,22]$. The moduli of Lagrangian submanifolds with topology $S^{1} \times \mathbb{R}^{2}$ was described by the zero locus

$$
\{A(x, p ; Q)=0\} \subset \hat{X} .
$$

Moreover, the image of a shifted conormal bundle to the unknot under the conifold transition described in Section 2.2 is exactly one of these Lagrangian fibers [4,23]. Hence, one might speculate 
that the moduli of Lagrangian submanifolds $L_{K} \subset X$ obtained from the conormal bundles of other knots $K \subset S^{3}$ are given by the Riemann surface

$$
\left\{A_{K}(x, p ; Q)=0\right\},
$$

where $A_{K}(x, p ; Q)$ is the augmentation polynomial of the knot $K$.

Aganagic and Vafa then conjecture that, for each choice of knot, there is a corresponding mirror $\hat{X}_{K}$ describing the moduli space of Lagrangian fibers with geometry $L_{K}$ along the singular locus, and that $\hat{X}_{K}$ is described by

$$
\hat{X}_{K}:=\left\{u v=A_{K}(x, p ; Q)\right\} .
$$

In addition to the apparent coincidence between the unknot's augmentation polynomial and the mirror $\hat{X}$, there are also physical arguments for this conjecture coming from the connections between topological string theory, Chern-Simons theory, and the HOMFLY polynomial $[11,12,21,24]$. The augmentation polynomial $A_{K}$ can be identified with the classical limit of the $Q$-deformed quantum A-polynomial, which satisfies an elimination condition on the Chern-Simons partition function in the presence of a Wilson loop coming from $K$. This Chern-Simons partition function can in turn be obtained from the colored HOMFLY polynomials of $K$, and is identified with the open Gromov-Witten generating function under the conifold transition.

For the purposes of computing genus-zero open Gromov-Witten invariants, the application of this conjecture is straightforward: as in Section 2.1, the open Gromov-Witten generating function is equated with the integral of a differential form:

$$
\sum_{d, w} K_{d, w} Q^{d} x^{w}=\int-\log p(x ; Q) \frac{d x}{x} .
$$

However, $p(x ; Q)$ is now determined by the equation

$$
A_{K}(x, p ; Q)=0
$$

The main difficulty is that $A_{K}(x, p ; Q)$ may be a high-order polynomial in $p$, so it is not always feasible to find analytic solutions for $p$. Even for torus knots, this can be an obstacle: for an $(r, s)$ torus knot,

$$
\operatorname{deg} p=\left(\begin{array}{c}
r+s \\
s
\end{array}\right), \quad \operatorname{deg} x=\frac{\operatorname{deg} p}{r+s}
$$

are the maximum degrees [21].

Fortunately, to compute $K_{d, w}$ for a given $d$ and $w$, only a series solution for $p$ is needed. Suppose that

$$
p(x ; Q)=\exp \left(-\sum_{n=0}^{\infty} W_{n}(Q) x^{n}\right),
$$

where $W_{n}(Q)$ is a polynomial in $Q$ for $n>0$, and $W_{0}(Q)$ determines the overall scaling of $p$. (Note that in all considered examples, $W_{0}(Q)=\log (Q)$, i.e., $\left.p(x ; Q) \approx 1 / Q+\cdots\right)$. Then, substitute this expression into

$$
A_{K}(x, p(x ; Q) ; Q)=0 .
$$

The resulting expression will be a series in $x$, which can be solved by recursively finding the coefficients $W_{n}(Q)$-in general, the coefficient of $x^{n}$ will be a polynomial function of $\left\{W_{k}(Q)\right\}_{k \leq n}$. The coefficients $W_{n}(Q)$ are related to the $K_{d, w}$ by

$$
W_{n}(Q)=\sum_{d} n K_{d, w} Q^{d}
$$


For completeness, the integer invariants $N_{d, w}$ can be similarly determined from the $K_{d, w}$ : Equation (7) can be re-written as

$$
N_{d, w}=K_{d, w}-\sum_{n \mid d \text { and } n \mid w} \frac{1}{n^{2}} N_{d / n, w / n}
$$

so by starting with $N_{0,1}=K_{0,1}$, successive $N_{d, w}$ can be solved for. The following section uses this method to compute $K_{d, w}$ and $N_{d, w}$ for two non-toric knots.

\section{Non-Toric Examples}

As compared with Atiyah-Bott localization, one advantage of computing open Gromov-Witten invariants from the augmentation polynomial is that this technique does not require that the Lagrangian $L$ is fixed by a torus action. Such Lagrangians can be obtained from the conormal bundles of non-toric knots. This section performs the computation of Section 2.3 for two non-toric knots: the $4_{1}$ (figure-eight) knot and the $5_{2}$ (three-twist) knot. In contrast to torus knots, these knots cannot be expressed as the link of the singularity $x^{r}-y^{s}=0$ in $S^{3}$ for any $r, s \in \mathbb{Z}$. For non-toric knots, it is not currently known how to directly compute the open Gromov-Witten invariants $K_{d, w}$ using localization. However, the invariants computed in the following examples have been checked to satisfy the integrality condition (7) for all $w \leq 8$ and arbitrary $d$, and the results may be compared against the colored HOMFLY polynomials in the symmetric representations.

\subsection{The $4_{1}$ (Figure-Eight) Knot}

The $4_{1}$ knot is the unique knot with crossing number 4 , and is a twist knot obtained from two half-twists. According to [11,23], the augmentation polynomial of the $4_{1}$ knot in framing 0 is

$$
\begin{aligned}
A_{K}(x, p, Q) & =p^{2}-Q p^{3}+\left(Q^{3} p^{5}-2 Q^{3} p^{4}+2 Q p-Q\right) x \\
& +\left(-Q^{5} p^{5}+2 Q^{4} p^{4}-2 Q^{3} p+Q^{2}\right) x^{2} \\
& +\left(Q^{5} p^{3}-Q^{5} p^{2}\right) x^{3} .
\end{aligned}
$$

Rescaling $x$ by $x \mapsto Q x$, one can obtain a series solution for $-\log p$ using the method of Section 2.3. The first few terms are

$$
\begin{aligned}
-\log p & =\log (Q)+\left(Q^{3}-2 Q^{2}+2 Q-1\right) x \\
& +\left(\frac{5 Q^{6}}{2}-8 Q^{5}+9 Q^{4}-9 Q^{2}+8 Q-\frac{5}{2}\right) x^{2}+\cdots
\end{aligned}
$$

The coefficients of this series solution determine the open Gromov-Witten invariants $K_{d, w}$ and corresponding integer invariants $N_{d, w}$. For $w \leq 4,0 \leq d \leq 3$, these invariants are listed in Tables 1 and 2. The integrality condition $N_{d, w} \in \mathbb{Z}$ has also been verified for all $w \leq 8$ and arbitrary $d$. Note that, for $w \leq 8, K_{d, w}=N_{d, w}=0$ for any $d>24$.

Table 1. $K_{d, w}$ for the $4_{1}$ knot.

\begin{tabular}{rrrrr}
\hline$K_{d, w}$ & $\boldsymbol{K}_{d, 1}$ & $\boldsymbol{K}_{d, 2}$ & $\boldsymbol{K}_{\boldsymbol{d}, 3}$ & $\boldsymbol{K}_{\boldsymbol{d}, 4}$ \\
\hline$K_{0, w}$ & -1 & $-\frac{5}{4}$ & $-\frac{28}{9}$ & $-\frac{165}{16}$ \\
$K_{1,3}$ & 2 & 4 & 14 & 60 \\
$K_{2, w}$ & -2 & $-\frac{9}{2}$ & -25 & -147 \\
$K_{3, w}$ & 1 & 0 & $\frac{173}{9}$ & 186 \\
\hline
\end{tabular}


Table 2. $N_{d, w}$ for the $4_{1}$ knot.

\begin{tabular}{rrrrr}
\hline$K_{\boldsymbol{d}, \boldsymbol{w}}$ & $\boldsymbol{K}_{\boldsymbol{d}, \mathbf{1}}$ & $\boldsymbol{K}_{\boldsymbol{d , \boldsymbol { 2 }}}$ & $\boldsymbol{K}_{\boldsymbol{d}, \mathbf{3}}$ & $\boldsymbol{K}_{\boldsymbol{d}, \mathbf{4}}$ \\
\hline$K_{0, w}$ & -1 & $-\frac{5}{4}$ & $-\frac{28}{9}$ & $-\frac{165}{16}$ \\
$K_{1,3}$ & 2 & 4 & 14 & 60 \\
$K_{2, w}$ & -2 & $-\frac{9}{2}$ & -25 & -147 \\
$K_{3, w}$ & 1 & 0 & $\frac{173}{9}$ & 186 \\
\hline
\end{tabular}

\subsection{The $5_{2}$ (Three-Twist) Knot}

The $5_{2}$ knot is a twist knot obtained from three half-twists, and is one of two knots with crossing number 5 (the other being the $(5,2)$ torus knot). The augmentation polynomial for the $5_{2}$ knot is

$$
\begin{aligned}
A_{K}(x, p, Q) & =Q^{2} p^{8}-Q p^{7}+x^{4}(-p+1) \\
& +\left(-Q^{3} p^{6}+2 Q^{2} p^{5}-Q p^{4}-2 Q p^{3}+3 Q p^{2}-3 p^{2}+4 p-2\right) x^{3} \\
& +\left(Q^{4} p^{8}-3 Q^{3} p^{7}-4 Q^{3} p^{6}+5 Q^{2} p^{6}+3 Q^{2} p^{5}\right. \\
& \left.+6 Q^{2} p^{4}-3 Q p^{5}-4 Q p^{4}+3 Q p^{3}-4 Q p^{2}-3 p^{3}+5 p^{2}-3 p+1\right) x^{2} \\
& +\left(-2 Q^{3} p^{8}+4 Q^{2} p^{7}+3 Q^{2} p^{6}-3 Q p^{6}-2 Q p^{5}-p^{4}+2 p^{3}-p^{2}\right) x .
\end{aligned}
$$

Rescaling $x$ by $x \mapsto x / Q$, the method of Section 2.3 gives a series solution for $-\log p$. The first few terms are

$$
\begin{aligned}
-\log p & =\log (Q)+\left(-Q^{4}+2 Q^{3}-Q\right) x \\
& +\left(\frac{11 Q^{8}}{2}-20 Q^{7}+23 Q^{6}-8 Q^{5}+2 Q^{4}-4 Q^{3}+\frac{3 Q^{2}}{2}\right) x^{2}+\cdots
\end{aligned}
$$

The corresponding $K_{d, w}$ and $N_{d, w}$ are obtained from the coefficients of this series solution. These invariants are listed in Tables 3 and 4 . As for the $4_{1}$ knot, the integrality condition $N_{d, w} \in \mathbb{Z}$ has also been checked for all $w \leq 8$, and it was again found that $K_{d, w}=N_{d, w}=0$ for any $w \leq 8$ and $d>24$.

Table 3. $K_{d, w}$ for the $5_{2}$ knot.

\begin{tabular}{rrrrr}
\hline$K_{d, w}$ & $K_{d, \mathbf{1}}$ & $K_{d, \mathbf{2}}$ & $K_{d, \mathbf{3}}$ & $\boldsymbol{K}_{\boldsymbol{d , 4}}$ \\
\hline$K_{0, w}$ & -1 & $\frac{3}{4}$ & $-\frac{10}{9}$ & $\frac{35}{16}$ \\
$K_{1,3}$ & 0 & -2 & 4 & -12 \\
$K_{2, w}$ & 2 & 1 & 0 & $\frac{27}{2}$ \\
$K_{3, w}$ & -1 & -4 & -12 & 8 \\
\hline
\end{tabular}

Table 4. $N_{d, w}$ for the $5_{2}$ knot.

\begin{tabular}{rrrrr}
\hline$N_{\boldsymbol{d}, \boldsymbol{w}}$ & $\boldsymbol{N}_{\boldsymbol{d , \mathbf { 1 }}}$ & $\boldsymbol{N}_{\boldsymbol{d}, \mathbf{2}}$ & $\boldsymbol{N}_{\boldsymbol{d}, \mathbf{3}}$ & $\boldsymbol{N}_{\boldsymbol{d}, \mathbf{4}}$ \\
\hline$N_{0, w}$ & -1 & 1 & -1 & 2 \\
$N_{1,3}$ & 0 & -2 & 4 & -12 \\
$N_{2, w}$ & 2 & 1 & 0 & 14 \\
$N_{3, w}$ & -1 & -4 & -12 & 8 \\
\hline
\end{tabular}

\section{Recovering the Augmentation Polynomial}

The augmentation polynomial conjecturally contains all of the open Gromov-Witten invariants $K_{d, w}$ for a given Lagrangian brane $L_{K}$. As seen in the previous section, open Gromov-Witten 
invariants can be extracted from the augmentation polynomial. For torus knots, the genus zero open Gromov-Witten invariants can also be computed directly via localization. The formula is:

Lemma 1. Suppose $(r, s)$ are coprime, $r>s>0$, and $w s \geq d>0$. Let $K$ be the $(r, s)$ torus $k n o t$, and $X, L_{K}$, and $K_{d, w}$ as above. Then,

$$
K_{d, w}=(-1)^{d+1}\left(\frac{\prod_{k=0}^{d-1}(w r-k)(w s-k)}{w d ! \prod_{k=1}^{d}(w r+w s-k)}\right)\left(\frac{\prod_{k=1}^{w s-1}\left(r+s-\frac{k}{w}\right)}{w \prod_{k=0}^{w s-1}\left(s-\frac{k}{w}\right)}\right),
$$

where $(r, s)$ are coprime, $r>s>0$ and $w s \geq d \geq 0$. For $d>w s, K_{d, w}=0$.

The proof of this lemma is postponed to the Appendix A.

This section describes a method for obtaining the augmentation polynomial $A_{K}$ when $K$ is a torus knot, and implements this method for two examples (the unknot, and the $(3,2)$ torus knot).

The idea is to use Equations (9) and (10) to obtain a system of linear equations on the coefficients of the augmentation polynomial. Write

$$
A_{K}(x, p ; Q)=\sum_{j, k} c_{j k} x^{j}(p(x ; Q))^{k},
$$

where $c_{j k}$ is a rational polynomial in $Q$. Recall that, for torus knots, the degrees of $x$ and $p$ are given by Equation (8). Let

$$
p(x ; Q)=\frac{1}{Q} \exp \left(-\sum_{d, w} w K_{d, w} Q^{d} x^{w}\right)
$$

and $W_{n}:=\sum_{d \geq 0} n K_{d, n} Q^{d}$. Then, the coefficient of $x^{n}$ in a series expansion of $(p(x ; Q))^{k}$ is $P_{n}(k) / Q^{k}$, where

$$
P_{n}(k):=\sum_{i_{1}+2 i_{2}+\cdots+n i_{n}=n}\left[\prod_{j=1}^{n} \frac{(-1)^{i_{j}}}{i_{j} \geq 0}\left(k W_{j}\right)^{i_{j}}\right],
$$

and $P_{0}(k)=1$. Substituting this expression into Equation (11) gives a power series in $x$ :

$$
\begin{aligned}
A_{K}(x, p ; Q) & =\sum_{j, k} c_{j k}\left(\frac{x^{j}}{Q^{k}}\right)\left(\sum_{n \geq 0} P_{n}(k) x^{n}\right) \\
& =\sum_{n \geq 0}\left(\sum_{j=0}^{n} \sum_{k \geq 0} \frac{P_{n-j}(k)}{Q^{k}} c_{j k}\right) x^{n} .
\end{aligned}
$$

In order for $p(x ; Q)$ to be a solution of $A_{K}(x, p ; Q)=0$, the coefficient of $x^{n}$ for all $n$ in the above expression must vanish. This gives a collection of linear equations in the $c_{j k}$ :

$$
\sum_{j=0}^{n} \sum_{k \geq 0} \frac{P_{n-j}(k)}{Q^{k}} c_{j k}=0,
$$

which can be solved to determine the $c_{j k}$ up to an overall rescaling of the augmentation polynomial. (Note that such rescalings do not affect the solutions $p(x ; Q)$, and hence do not affect the Gromov-Witten invariants). The following two examples implement this procedure for the unknot in framing 0 and the $(3,2)$ torus knot in framing 6 . For both knots, this method recovers the expected augmentation polynomial. However, for the $(3,2) \mathrm{knot}$, due to computational complexity, some simplifying assumptions about the values of the $c_{j k}$ are made. 


\subsection{The Unknot}

For the unknot, $\operatorname{deg} x=1, \operatorname{deg} p=1$, so there are four coefficients to solve for: $c_{00}, c_{01}, c_{10}$, and $c_{11}$. Here, the $W_{n}$ have a simple expression:

$$
W_{n}=\frac{1}{n}\left(Q^{n}-1\right)
$$

The first four linear equations from Equation (12) are:

$$
\begin{aligned}
c_{00}+\frac{1}{Q} c_{01} & =0, & & (n=0), \\
\frac{1}{Q}(1-Q) c_{01}+c_{10}+\frac{1}{Q} c_{11} & =0, & & (n=1), \\
\frac{1}{Q}(1-Q) c_{01}+\frac{1}{Q}(1-Q) c_{11} & =0, & & (n=2,3) .
\end{aligned}
$$

(The $n=2$ and $n=3$ equations both simplify to the same expression). With $c_{00}$ as the free variable, these equations become

$$
c_{01}=-Q c_{00}, \quad c_{10}=-Q c_{00}, \quad c_{11}=Q c_{00} .
$$

Normalizing to $c_{00}=1$ yields the expected augmentation polynomial of the unknot in framing 0 :

$$
A_{K}(x, p ; Q)=1-Q x-Q p+Q x p .
$$

\subsection{The $(3,2)$ Torus Knot}

The augmentation polynomial of the $(3,2)$ torus knot is

$$
\begin{aligned}
A_{K}(x, p ; Q) & =1-Q p+\left(Q^{5} p^{3}-Q^{5} p^{4}+2 Q^{5} p^{5}-2 Q^{6} p^{5}-Q^{6} p^{6}+Q^{7} p^{7}\right) x \\
& +\left(Q^{10} p^{10}-Q^{10} p^{9}\right) x^{2}
\end{aligned}
$$

For the $(3,2)$ torus $k \operatorname{knot}, \operatorname{deg} p=10$ and $\operatorname{deg} x=2$. Thus, $c_{j k}=0$ for all $j>2$ and $k>10$. Note that, in general, one would need to consider at least $(\operatorname{deg} p+1)(\operatorname{deg} x+1)$ equations to determine $c_{j k}$. Due to the increasing complexity of the equations involved, in this example, the following simplifying assumptions (These assumptions are made to simplify the computation presented here and in principle are not needed.) will be made: $c_{0 k}=0$ for $k>1, c_{10}=c_{11}=c_{12}=0$, $c_{18}=c_{19}=c_{1,10}=0$, and $c_{2 k}=0$ for $k<9$. Thus, the remaining nine "unknown" coefficients are $c_{00}$, $c_{01}, c_{13}, c_{14}, c_{15}, c_{16}, c_{17}, c_{29}$, and $c_{2,10}$. The first three equations from Equation (12) are:

$$
\begin{array}{r}
c_{00}+\frac{1}{Q} c_{01}=0, \\
\left(2 Q^{-1}-3+Q\right) c_{01}+Q^{-3} c_{13}+Q^{-4} c_{14}+Q^{-5} c_{15}+Q^{-6} c_{16}+Q^{-7} c_{17}=0 \\
\left(23 Q^{-1}-62+59 Q-23 Q^{2}+3 Q^{3}\right) c_{01}+\left(3 Q^{-1}-9 Q^{-2}+6 Q^{-3}\right) c_{13} \\
+\left(4 Q^{-2}-12 Q^{-3}+8 Q^{-4}\right) c_{14}+\left(5 Q^{-3}-15 Q^{-4}+10 Q^{-5}\right) c_{15} \\
+\left(6 Q^{-4}-18 Q^{-5}+12 Q^{-6}\right) c_{16}+\left(7 Q^{-5}-21 Q^{-6}+14 Q^{-7}\right) c_{17} \\
+Q^{-9} c_{29}+Q^{-10} c_{2,10}=0 .
\end{array}
$$


(For brevity, the remaining equations are omitted.) Solving for the $c_{i j}$ (with $c_{00}$ as the free variable), one finds

$$
\begin{aligned}
& c_{01}=-Q c_{00}, \quad c_{13}=Q^{5} c_{00}, \quad c_{14}=-Q^{5} c_{00}, \quad c_{15}=\left(2 Q^{5}-2 Q^{6}\right) c_{00}, \\
& c_{16}=-Q^{6} c_{00}, \quad c_{17}=Q^{7} c_{00}, \quad c_{29}=-Q^{10} c_{00}, \quad c_{2,10}=Q^{10} c_{00} .
\end{aligned}
$$

By normalizing to $c_{00}=1$, the expected coefficients of the augmentation polynomial,

$$
\begin{aligned}
& c_{01}=-Q, \quad c_{13}=Q^{5}, \quad c_{14}=-Q^{5}, \quad c_{15}=2 Q^{5}-2 Q^{6}, \\
& c_{16}=-Q^{6}, \quad c_{17}=Q^{7}, \quad c_{29}=-Q^{10}, \quad c_{2,10}=Q^{10} \text {, }
\end{aligned}
$$

are obtained.

\section{Conclusions}

Because of the difficulties involved in the computation of open Gromov-Witten invariants, a comparison of the technique described here against direct calculation is only currently possible in the torus knot case. A comparison for the non-toric case will require new techniques in enumerative geometry to verify. However, in all cases considered here, the computed open invariants are shown to satisfy a strong integrality condition expected of Gromov-Witten invariants. This can be seen as evidence for Aganagic and Vafa's conjecture (Conjecture 1). Moreover, in the torus knot case, the corresponding open Gromov-Witten invariants have been computed explicitly in Lemma 1, so a direct comparison is possible-and here agreement is seen, both by comparing the Gromov-Witten invariants against series terms obtained from the augmentation polynomial, and by using the Gromov-Witten invariants to produce the augmentation polynomial. In the latter case, this provides a generic algorithm for computing the augmentation polynomial of any $(r, s)$ torus knot.

Acknowledgments: This work was self-funded by the author.

Conflicts of Interest: The author declares no conflict of interest. The founding sponsors had no role in the design of the study; in the collection, analyses, or interpretation of data; in the writing of the manuscript, and in the decision to publish the results.

\section{Appendix A. Proof of Lemma 1}

This appendix provides a proof of Lemma 1 from Section 4 . The statement of the Lemma is:

Lemma A1. Suppose $(r, s)$ are coprime, $r>s>0$, and $w s \geq d>0$. Let $K$ be the $(r, s)$ torus knot, and $X$, $L_{K}$, and $K_{d, w}$ as above. Then,

$$
K_{d, w}=(-1)^{d+1}\left(\frac{\prod_{k=0}^{d-1}(w r-k)(w s-k)}{w d ! \prod_{k=1}^{d}(w r+w s-k)}\right)\left(\frac{\prod_{k=1}^{w s-1}\left(r+s-\frac{k}{w}\right)}{w \prod_{k=0}^{w s-1}\left(s-\frac{k}{w}\right)}\right),
$$

where $(r, s)$ are coprime, $r>s>0$ and $w s \geq d \geq 0$. For $d>w s, K_{d, w}=0$.

Proof. The idea of the proof is to incorporate the contribution from the open disk into the generating function for the closed Gromov-Witten invariants on $X=\mathcal{O}_{\mathbb{P}^{1}}(-1,-1)$. The authors of [10] computed the contribution of the open disk Gromov-Witten invariants associated with the $(r, s)$ knot for winding-1 (that is, the case $d=0, w=1$ ). The calculation in [10] readily generalizes to arbitrary winding as

$$
K_{0, w}=-\left(\frac{1}{w}\right)\left(\frac{\prod_{k=1}^{w s-1}\left(r+s-\frac{k}{w}\right)}{w \prod_{k=0}^{w s-1}\left(s-\frac{k}{w}\right)}\right),
$$


so what remains is to compute the contribution from the closed part (i.e., non-disk components) of the Gromov-Witten invariant. The computation from the closed part can be obtained from the calculation of the corresponding closed Gromov-Witten invariant [25]. One way to obtain a generating function for the closed terms is from Givental's J function.

Closed Gromov-Witten invariants appear as terms in Givental's $J$ function [26], and the map on quantum cohomology $J_{X}: H^{*}(X ; \mathbb{Q}) \longrightarrow H^{*}(X ; \mathbb{Q})(z)$ is given by

$$
J_{X}(\gamma)=z+\gamma+\sum_{n=0}^{\infty} \sum_{d \in H_{2}(X ; \mathbb{Z})} \frac{Q^{d}}{n !}\left\langle\gamma^{n}, \frac{T^{\alpha}}{z-\psi}\right\rangle_{0, d} T_{\alpha},
$$

where $T^{\alpha}$ is a basis for the cohomology of $X, T_{\alpha}$ is the dual basis with respect to the Poincare pairing, and

$$
\left\langle\gamma^{n}, \frac{T^{\alpha}}{z-\psi}\right\rangle_{0, d}=\sum_{k=0}^{\infty} z^{-(k+1)}\left\langle\gamma^{n}, \tau_{k} T^{\alpha}\right\rangle_{0, d}
$$

is a power series of gravitational descendant closed Gromov-Witten invariants.

As described in $[27,28]$, a generating function for one-boundary-component, genus-zero open Gromov-Witten invariants can be obtained from the composition of Givental's J-function with a disk term:

$$
\sum_{d=0}^{\infty} K_{d, w} Q^{d}=\left.\Delta_{X, L} \circ J_{X}\left(\phi_{p}\right)\right|_{z=1 / w},
$$

and $J_{X}$ can be obtained from $J_{\mathbb{P}^{1}}$ by a twisting procedure. From [29],

$$
J_{\mathbb{P}^{1}}=e^{\left(t_{0}+t_{2} H\right) / z} \sum_{d=0}^{\infty} \frac{1}{((1+H)(2+H) \cdots(d+H))^{2}} Q^{d},
$$

where $H$ is the hyperplane section on $\mathbb{P}^{1}$ and $t_{0}, t_{2}$ are coordinates for the $\{\mathbf{1}, p\}$ basis for $H^{*}\left(\mathbb{P}^{1}\right)$. Gromov-Witten invariants on $X$ are related to Gromov-Witten invariants on $\mathbb{P}^{1}$ by

$$
\int_{\left[\overline{\mathcal{M}}_{0,0}(X ; d)\right]^{v i r}} 1=\int_{\left[\overline{\mathcal{M}}_{0,0}\left(\mathbb{P}^{1} ; d\right)\right]^{v i r}} c_{2 d-2}\left(\mathcal{F}_{d}\right),
$$

where $\mathcal{F}_{d}$ is the obstruction sheaf whose fiber at each point $(\Sigma, f)$ is

$$
H^{1}\left(\Sigma, f^{*} \mathcal{O}_{\mathbb{P}^{1}}(-1,-1)\right)
$$

(see [5]). The right-hand side of Equation (A1) is sometimes referred to as a Gromov-Witten invariant "twisted" by $\mathcal{F}_{d}$. In [30], the authors have worked out a general procedure for obtaining the twisted $J$-function $J_{M}^{t w}$ of a target space $M$, defined by

$$
J_{M}^{t w}(\gamma, z):=z+\gamma+\sum_{n \geq 0} \sum_{d} \frac{Q^{d}}{n !}\langle\overbrace{\gamma, \gamma, \ldots, \gamma}^{n \text { times }}, \frac{T^{\alpha}}{z-\psi}\rangle_{0, d}^{t w} T_{\alpha}
$$

where $T^{\alpha}$ is a basis for $H^{*}(M)$, and

$$
\left\langle\gamma_{1}, \ldots, \gamma_{n}, \frac{1}{z-\psi}\right\rangle_{0, d}^{t w}:=\sum_{k \geq 0} z^{-k-1} \int_{\left[\overline{\mathcal{M}}_{0, n+1}(M ; d)\right]^{j i r}} c\left(\mathcal{F}_{d}\right)\left(\prod_{i} e v_{i}^{*}\left(\gamma_{i}\right) \psi_{i}^{k}\right)
$$

is a power series of "twisted" descendant Gromov-Witten invariants. When $c\left(\mathcal{F}_{d}\right)$ is trivial, this reduces to the usual untwisted Gromov-Witten invariants. The next steps are to apply the twisted formula of [30] for $X=\mathcal{O}_{\mathbb{P}^{1}}(-1,-1)$, and then localize the resulting expression. 
In a forthcoming paper [31], the author has carefully worked out an expression for the localized form of the twisted $J$-function associated with the resolved conifold in terms of Gauss's hypergeometric function ${ }_{2} F_{1}(a, b ; c ; z)$. Recall that this is the hypergeometric function defined by

$$
{ }_{2} F_{1}(a, b, ; c ; z)=\sum_{n=0}^{\infty} \frac{(a)_{n}(b)_{n}}{(c)_{n}} \frac{z^{n}}{n !},
$$

where

$$
(q)_{n}= \begin{cases}1, & n=0, \\ \prod_{k=0}^{n-1}(q+k), & n>0 .\end{cases}
$$

According to [31], the localized $J$-function of $X$ is

$$
J_{X}\left(t_{0}, t_{2}, z\right)=z e^{\left(t_{0}+t_{2} \lambda_{\tilde{\xi}}\right) / z}{ }_{2} F_{1}\left(-\frac{\lambda_{x}}{z},-\frac{\lambda_{y}}{z} ; 1+\frac{\lambda_{\xi}}{z} ; e^{t_{2}}\right),
$$

where in the $\left\{\mathbf{1}_{X}, p\right\}$ basis for the $S^{1}$-equivariant cohomology $H_{S^{1}}(X), t=t_{0} \mathbf{1}_{X}+t_{2} p$, and $\lambda_{\xi}, \lambda_{x}$, and $\lambda_{y}$ are the weights of the $S^{1}$ action on $X$. In this case, $\lambda_{x}=s, \lambda_{y}=r$, and $\lambda_{\xi}=-r-s$. Note that $e^{t_{2}}=Q$. Then,

$$
J_{X}=\left(e^{w t_{0}} Q^{-w(r+s)}\right) z \sum_{d=0}^{\infty} \frac{\left(-\frac{s}{z}\right)_{d}\left(-\frac{r}{z}\right)_{d}}{\left(1-\frac{r+s}{z}\right)_{d}} \frac{Q^{d}}{d !} .
$$

After sending $t_{0} \mapsto 0$, the $Q^{-w(r+s)}$ factor can be discarded because its only effect is to shift the powers of $Q$. Specializing to $z=\frac{1}{w}, \Delta_{X, L} \circ J_{X}$ becomes

$$
\begin{aligned}
\left.\Delta_{X, L} \circ J_{X}\left(\phi_{p}\right)\right|_{z=\frac{1}{w}} & =D_{X, L} \sum_{d=0}^{\infty} \frac{Q^{d}}{w d !} \frac{\prod_{k=0}^{d-1}(k-w s)(k-w r)}{\prod_{k=0}^{d-1}(k+1-w r-w s)} \\
& =\left(-\frac{\prod_{k=1}^{w s-1}\left(r+s-\frac{k}{w}\right)}{w \prod_{k=0}^{w s-1}\left(s-\frac{k}{w}\right)}\right)\left[\sum_{d=0}^{\infty}\left((-1)^{d} \frac{\prod_{k=0}^{d-1}(w s-k)(w r-k)}{w d ! \prod_{k=1}^{d}(w r+w s-k)}\right) Q^{d}\right] .
\end{aligned}
$$

The coefficient of $Q^{d}$ in this expression is $K_{d, w}$. This completes the proof.

\section{References}

1. Candelas, P.; de la Ossa, X.C.; Green, P.S.; Parkes, L. A pair of Calabi-Yau manifolds as an exactly soluble superconformal theory. Nuclear Phys. B 1991, 359, 21-74.

2. Witten, E. Mirror manifolds and topological field theory. In Essays on Mirror Manifolds; International Press: Hong Kong, China, 1992; pp. 120-158.

3. Gopakumar, R.; Vafa, C. On the gauge theory/geometry correspondence. Adv. Theor. Math. Phys. 1999, 3, 1415-1443.

4. Ooguri, H.; Vafa, C. Knot invariants and topological strings. Nuclear Phys. B 2000, 577, 419-438.

5. Kontsevich, M. Enumeration of rational curves via torus actions. In The Moduli Space of Curves (Texel Island, 1994); Birkhäuser Boston: Boston, MA, USA, 1995; Volume 129, pp. 335-368.

6. Graber, T.; Pandharipande, R. Localization of virtual classes. Invent. Math. 1999, 135, 487-518.

7. Chiang, T.M.; Klemm, A.; Yau, S.T.; Zaslow, E. Local mirror symmetry: Calculations and interpretations. Adv. Theor. Math. Phys. 1999, 3, 495-565.

8. Klemm, A.; Zaslow, E. Local mirror symmetry at higher genus. arXiv 1999, arXiv:hep-th/9906046.

9. Witten, E. Quantum field theory and the Jones polynomial. Commun. Math. Phys. 1989, 121, 351-399.

10. Diaconescu, D.E.; Shende, V.; Vafa, C. Large $N$ duality, Lagrangian cycles, and algebraic knots. Commun. Math. Phys. 2013, 319, 813-863.

11. Aganagic, M.; Vafa, C. Large N duality, mirror symmetry, and a Q-deformed A-polynomial for knots. arXiv 2012, arXiv:1204.4709 [hep-th]. 
12. Aganagic, M.; Ekholm, T.; Ng, L.; Vafa, C. Topological strings, D-model, and knot contact homology. Adv. Theor. Math. Phys. 2014, 18, 827-956.

13. $\mathrm{Ng}$, L. A topological introduction to knot contact homology. arXiv 2012, arXiv:1210.4803 [math.GT].

14. Strominger, A.; Yau, S.T.; Zaslow, E. Mirror symmetry is T-duality. Nuclear Phys. B 1996, 479, $243-259$.

15. Aganagic, M.; Vafa, C. Mirror symmetry, D-branes and counting holomorphic discs. arXiv 2000, arXiv:hep-th/0012041.

16. Hori, K.; Vafa, C. Mirror symmetry. arXiv 2000, arXiv:hep-th/0002222.

17. Aganagic, M.; Klemm, A.; Vafa, C. Disk instantons, mirror symmetry and the duality web. Z. Naturforsch. A 2002, 57, 1-28.

18. Katz, S.; Liu, C.C.M. Enumerative geometry of stable maps with Lagrangian boundary conditions and multiple covers of the disc. Adv. Theor. Math. Phys. 2001, 5, 1-49.

19. Labastida, J.; Mariño, M.; Vafa, C. Knots, links and branes at large N. J. High Energy Phys. 2001, 2000, doi:10.1088/1126-6708/2000/11/007.

20. Brini, A.; Eynard, B.; Mariño, M. Torus knots and mirror symmetry. Ann. Henri Poincaré 2012, 13, 1873-1910, doi:10.1007/s00023-012-0171-2.

21. Jockers, H.; Klemm, A.; Soroush, M. Torus knots and the topological vertex. Lett. Math. Phys. 2014, 104, 953-989.

22. Ekholm, T.; Etnyre, J.; Ng, L.; Sullivan, M. Knot contact homology. Geom. Topol. 2013, 17, 975-1112, doi:10.2140/gt.2013.17.975.

23. Gu, J.; Jockers, H.; Klemm, A.; Soroush, M. Knot invariants from topological recursion on augmentation varieties. Commun. Math. Phys. 2015, 336, 987-1051, doi:10.1007/s00220-014-2238-z.

24. Neitzke, A.; Walcher, J. background independence and the open topological string wavefunction. arXiv 2007, arXiv:0709.2390 [hep-th].

25. Graber, T.; Zaslow, E. Open-string Gromov-Witten invariants: calculations and a mirror "theorem". In Orbifolds in Mathematics and Physics (Madison, WI, 2001); American Mathematical Society: Providence, RI, USA, 2002; Volume 310, pp. 107-121.

26. Givental, A. Equivariant Gromov-Witten invariants. Internat. Math. Res. Notices 1996, 1996, 613-663.

27. Brini, A.; Cavalieri, R. Open orbifold Gromov-Witten invariants of $\left[\mathbb{C}^{3} / \mathbb{Z}_{n}\right]$ : Localization and Mirror Symmetry. arXiv 2010, arXiv:1007.0934 [math.AG].

28. Brini, A. Open topological strings and integrable hierarchies: remodeling the A-model. Commun. Math. Phys. 2012, 312, 735-780.

29. Cox, D.A.; Katz, S. Mirror symmetry and algebraic geometry. In Mathematical Surveys and Monographs; American Mathematical Society: Providence, RI, USA, 1999; Volume 68.

30. Coates, T.; Corti, A.; Iritani, H.; Tseng, H.H. Computing genus-zero twisted Gromov-Witten invariants. Duke Math. J. 2009, 147, 377-438.

31. Brini, A. (Imperial College London); Personal communication, 2016.

(C) 2017 by the author. Licensee MDPI, Basel, Switzerland. This article is an open access article distributed under the terms and conditions of the Creative Commons Attribution (CC BY) license (http://creativecommons.org/licenses/by/4.0/). 